\title{
Time-reversed ultrasonically encoded (TRUE) focusing for deep-tissue optogenetic modulation
}

Joshua Brake, Haowen Ruan, J. Elliott Robinson, Yan Liu, Viviana Gradinaru, et al.

Joshua Brake, Haowen Ruan, J. Elliott Robinson, Yan Liu, Viviana Gradinaru, Changhuei Yang, "Time-reversed ultrasonically encoded (TRUE) focusing for deep-tissue optogenetic modulation," Proc. SPIE 10502, Adaptive Optics and Wavefront Control for Biological Systems IV, 1050210 (23 February 2018); doi: 10.1117/12.2288331

SPIE. Event: SPIE BiOS, 2018, San Francisco, California, United States 


\title{
Time-reversed ultrasonically encoded (TRUE) focusing for deep-tissue optogenetic modulation
}

\author{
Joshua Brake ${ }^{\mathrm{a}}$, Haowen Ruan ${ }^{\mathrm{a}}$, J. Elliott Robinson ${ }^{\mathrm{b}}$, Yan Liu ${ }^{\mathrm{a}}$, Viviana Gradinaru ${ }^{\mathrm{b}}$, Changhuei Yang ${ }^{\mathrm{a}}$ \\ ${ }^{a}$ Dept. of Electrical Engineering, California Institute of Technology, 1200 E. California Blvd., \\ Pasadena, CA 91125, USA \\ ${ }^{b}$ Division of Biology and Biological Engineering, California Institute of Technology, Pasadena, CA \\ 91125, USA.
}

\begin{abstract}
The problem of optical scattering was long thought to fundamentally limit the depth at which light could be focused through turbid media such as fog or biological tissue. However, recent work in the field of wavefront shaping has demonstrated that by properly shaping the input light field, light can be noninvasively focused to desired locations deep inside scattering media. This has led to the development of several new techniques which have the potential to enhance the capabilities of existing optical tools in biomedicine. Unfortunately, extending these methods to living tissue has a number of challenges related to the requirements for noninvasive guidestar operation, speed, and focusing fidelity. Of existing wavefront shaping methods, time-reversed ultrasonically encoded (TRUE) focusing is well suited for applications in living tissue since it uses ultrasound as a guidestar which enables noninvasive operation and provides compatibility with optical phase conjugation for high-speed operation. In this paper, we will discuss the results of our recent work to apply TRUE focusing for optogenetic modulation, which enables enhanced optogenetic stimulation deep in tissue with a 4-fold spatial resolution improvement in 800-micron thick acute brain slices compared to conventional focusing, and summarize future directions to further extend the impact of wavefront shaping technologies in biomedicine.
\end{abstract}

Keywords: wavefront shaping, optogenetics, brain, time-reversed ultrasonically encoded (TRUE) focusing, timereversal, scattering, biophotonics, optical phase conjugation

\section{INTRODUCTION}

Focusing light deep into biological tissue has been an important area of research in optics for many years. Using light to interrogate biological systems has many advantages since optical methods can be noninvasive, nonionizing, exploit a wide range of contrast mechanisms, and support excellent temporal and spatial resolutions. However, one of the overriding challenges for optical imaging is its limited penetration depth in biological tissue. In contrast to other imaging modalities such as MRI or X-ray which can penetrate through centimeter thick biological structures, high-resolution optical imaging can conventionally be achieved only at superficial depths on the order of several hundred micrometers. This is due to the strong refractive index heterogeneity of biological tissue in the optical portion of the electromagnetic spectrum which causes light to be strongly scattered by tissue as it propagates ${ }^{1}$.

Traditionally, methods have been developed to deal with the strong scattering nature of tissue by exploiting the fact that longer wavelengths (e.g., in the near-infrared and infrared regime) are in general much less strongly scattered by biological tissue. For approaches such as multi-photon microscopy, this enables penetration depths 2-10 times greater than those available using single photon approaches ${ }^{2}$. However, even multi-photon microscopy is limited by its efficiency and maximum penetration depths which are on the order of a few millimeters. This is a consequence of not directly addressing the problem of scattering.

Other classes of optical technologies take a different approach to deal with scattering. Whereas typical microscopy techniques such as confocal or multi-photon microscopy treat scattered light as noise and try to gate it out, techniques such as diffuse optical tomography (DOT) try to capture and use the scattered light to infer certain characteristics of the tissue using a model of the scattering process. Other methods exploit additional contrast mechanisms such as the acoustic signal generated by light absorption that is used in photoacoustic tomography (PAT) to generate 3-dimensional images of optical absorption by collecting and processing the emitted ultrasonic waves. 
However, these methods also do not treat the underlying fundamental issue of light scattering. In principle, the process of light scattering is a deterministic process which is dictated by the composition and arrangement of the scattering components inside the scattering medium. In short, this means that if the composition of the scattering medium is known, the scattering process can be exactly predicted by the physics of wave propagation. Furthermore, the deterministic nature of scattering can be exploited without having to completely determine the physical composition of the scattering medium.

Following the demonstration in 2007 by Vellekoop et al. ${ }^{3}$ that the addition of a scattering medium in an optical system can actually enhance the ability to form a strong optical focus, many additional techniques were developed to focus light through or inside scattering media. Of particular interest are those developed to focus light inside scattering media instead of through them since these are more directly applicable for practical bioimaging and light delivery. These methods require a guidestar mechanism inside the medium to tag light passing through a location of interest. Popular guidestar mechanisms include fluorescent ${ }^{4}$, kinetic $^{5,6}$, photoacoustic ${ }^{7-10}$, ultrasonic ${ }^{11-14}$, ultrasound microbubble ${ }^{15}$, and magnetic particle ${ }^{16}$ based strategies. Of these, the ultrasound guidestar used in time-reversed ultrasonically encoded (TRUE) focusing ${ }^{12}$ offers the unique benefit of being noninvasive and freely addressable thus making it well suited for focusing inside biological systems.

Optogenetics is one developing area of research in biomedicine where noninvasive light delivery deep inside tissue can be of great use ${ }^{17}$. By genetically encoding light sensitive transmembrane proteins, neural activity can be excited or inhibited. However, current state of the art methods to deliver light to activate neurons of interest in regions of the brain below the superficial layers mainly rely on the implantation of optical elements such as optical fibers or gradient refractive index (GRIN) lenses ${ }^{18}$. While these enable much deeper penetration depths by physically channeling the light inside a waveguide structure, they are invasive in nature which can lead to damage and perturbation of the neural system being studied. In this paper, we describe the development and implementation of an integrated TRUE focusing and patch clamp electrophysiology system. After demonstrating the ability to focus through 2-mm-thick living brain tissue with the TRUE focusing system, we perform patch clamp recordings of optogenetically evoked photocurrents in $800-\mu \mathrm{m}$-thick brain tissue slices and demonstrate that TRUE focusing can enable a 4-fold improvement in the resolution of optogenetic modulation ${ }^{19}$. This represents the first demonstration of TRUE focusing in living brain tissue and is an important step in the translation of wavefront shaping tools for practical applications such as optogenetics in vivo.

\section{METHODOLOGY}
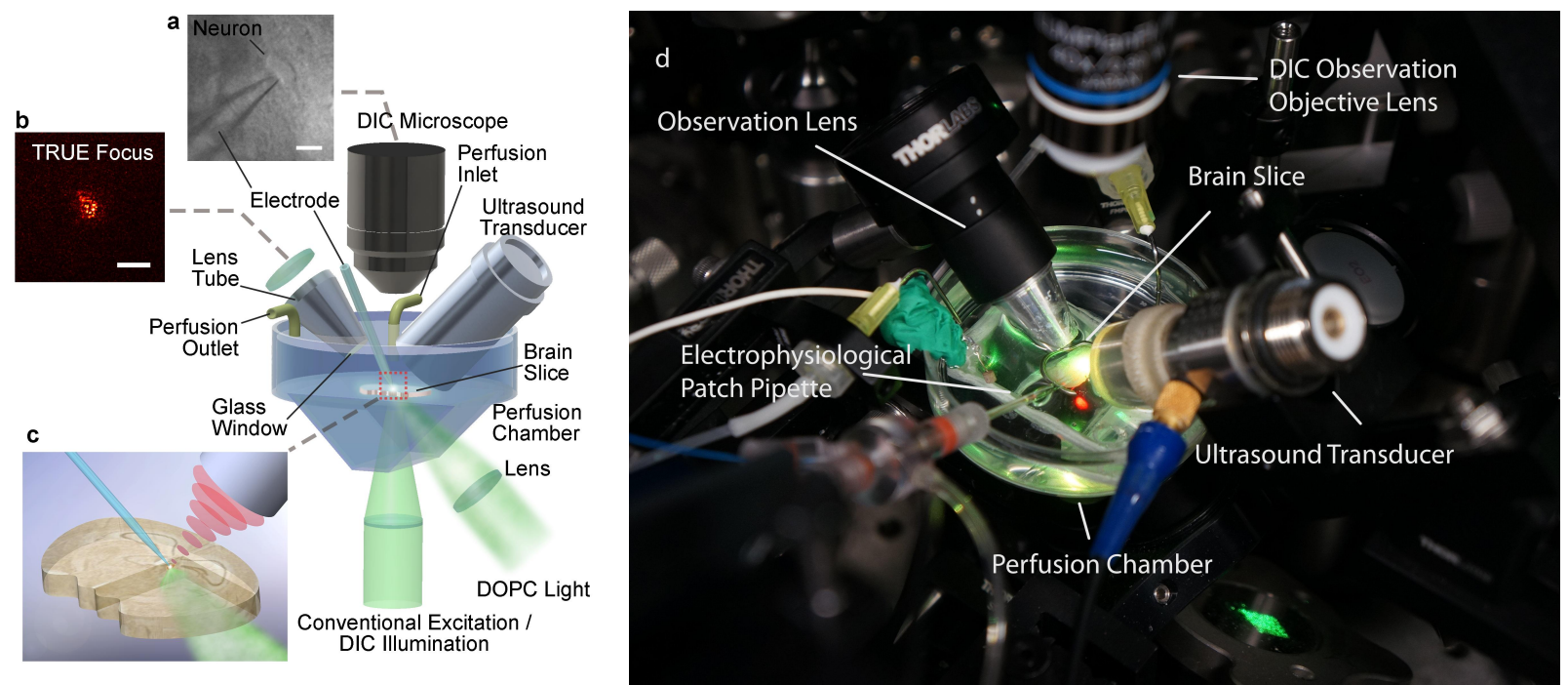

Figure 1. Diagram of the experimental setup. (a) A custom built DIC microscope is used to visualize and patch the neuron of interest. (b) The TRUE focusing procedure is then used to focus light onto the neuron. (c) A close-up visualization of the patch pipette, ultrasound focus, and TRUE focus. Scale bars, $20 \mu \mathrm{m}$ in (a) and $50 \mu \mathrm{m}$ in (b). (d) A photo of the experimental setup with annotations. Reproduced and modified from Reference 19. 
Fig. 1 shows a 3D schematic (Fig. 1(a-c)) and photograph (Fig. 1(d)) of the main portion of the experimental setup. A custom-built differential interference contrast (DIC) microscope is used to visualize the superficial layers of the acute brain slice and a glass electrode is used to patch clamp an individual neuron (Fig. 1(a)). Then, the DIC microscope objective is translated out of the chamber and the lens tube and ultrasound transducer are translated into the chamber, with the ultrasound focus located at the tip of the patch pipette electrode. Using the TRUE focusing procedure, an optical focus is formed on the neuron and used to elicit optogenetically induced signals which are monitored in either current or voltage clamp mode using the electrode (Fig. 1(b-c)).

A simplified schematic of the TRUE focusing system operation for optogenetic modulation is shown in Fig. 2. The TRUE focusing procedure consists of two steps, recording (Fig. 2(a1)) and playback (Fig. 2(a2)). In the recording step, a pulse of ultrasound $(50 \mathrm{MHz})$ is focused to the desired focusing location while a pulse of light from the probe beam (532 $\mathrm{nm}$ ) is sent into the tissue sample. The pulses are timed so that the light pulse passes through the tissue while the ultrasound is at the focus of the acoustic lens. Because the acoustic properties of brain tissue are relatively homogenous, the ultrasound can be tightly focused with conventional acoustic focusing techniques. However, the strong optical heterogeneity of the tissue causes light to be strongly scattered. After the light passes through the tissue, the scattered light field is measured using the camera in the digital optical phase conjugation (DOPC) system. Then, the conjugate phase map of the light field is calculated and displayed on the spatial light modulator (SLM) of the DOPC system and a blank beam is reflected off the SLM to form the playback beam. This beam passes through the tissue, and according to the principle of time-reversal, passes through the location of the ultrasound focus in the recording step, forming an optical focus at that location.

Fig. 2(b) shows the setup used to measure the conventional focusing of light through the brain slice, with light focused through the tissue from below and the scattered light profile measured from above. Results from focusing light with conventional and TRUE focusing through a variety of brain slice thicknesses are shown in Fig 2(c). As the tissue becomes thicker, the conventional focus diffuses and after a thickness of $1000 \mu \mathrm{m}$, no recognizable focus profile can be distinguished within the $580 \times 580-\mu \mathrm{m}^{2}$ field of view. In contrast, because the TRUE focusing technique shapes the wavefront to counteract the effects of scattering, a clear optical focus at the resolution of the ultrasound focus $(\sim 30 \mu \mathrm{m})$ can be maintained through the tissue regardless of its thickness. The full widths at half maximum (FWHM) of the focus profiles across the series of tissue thicknesses are shown in Fig. 2(d).

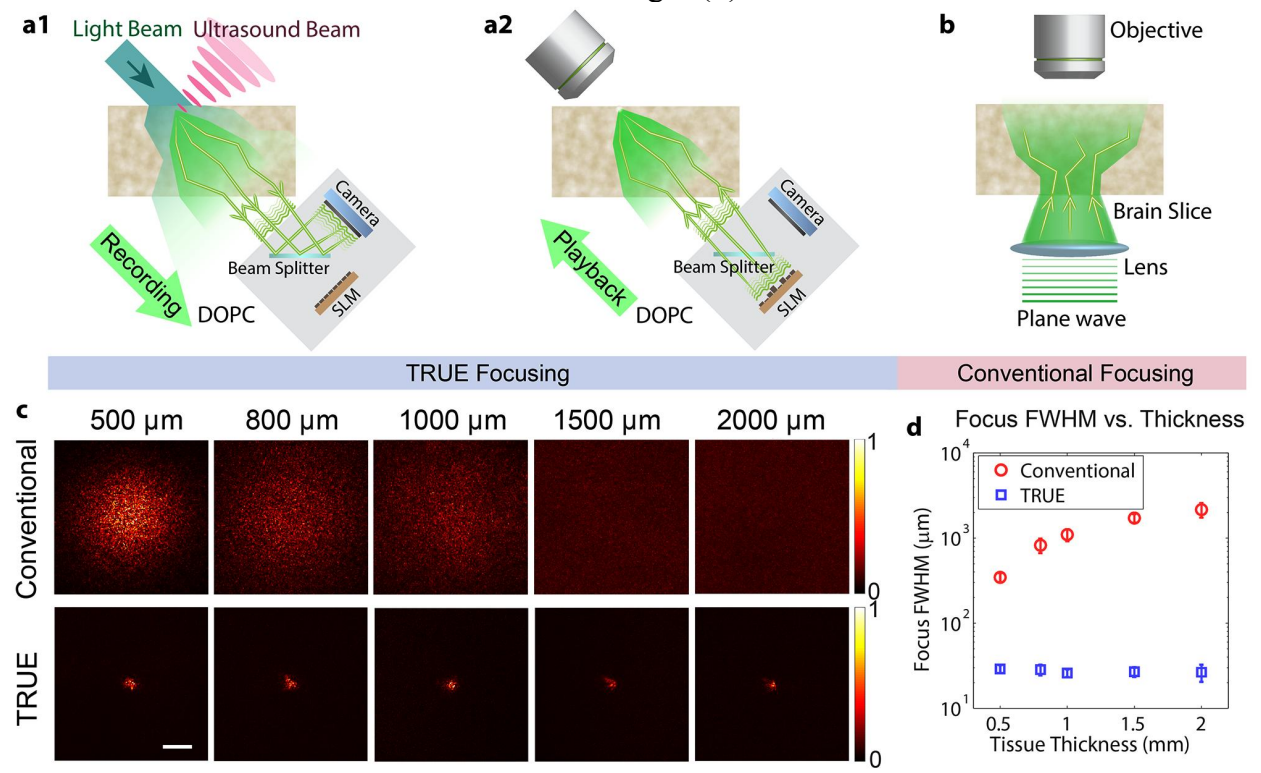

Figure 2. (a) The procedure to focus light through the brain slice onto its top surface consists of two steps: recording (a1) and playback (a2). (b) The setup used to measure the light intensity distribution on the top of the brain slice when using a conventional lens to focus through the brain slice. (c) Images of the conventional and TRUE focus for a selection of brain slice thicknesses from $500-2000 \mu \mathrm{m}$. (d) The full width at half maximum (FWHM) focal spots size for the conventional and TRUE foci. Error bars indicate the standard deviation of five measurements taken at different locations in the tissue. Scale bar, $100 \mu \mathrm{m}$. Reproduced from Reference 19. 


\section{RESULTS}

The results from optogenetic experiments with TRUE focusing are displayed in Fig. 3. First, the adeno-associated viral vector carrying the bReaChES transgene ${ }^{20}$ (AAV-DJ-CaMKII-bReaChES-TS-YFP) was injected into the medial prefrontal cortex (mPFC) of C57Bl/6J mice. After waiting 4 weeks for the mice to recover from the surgery and for the transgene to be expressed, we prepared acute brain slices for testing. Animal husbandry and all experimental procedures involving animal subjects were approved by the Institutional Animal Care and Use Committee (IACUC) and by the Office of Laboratory Animal Resources at the California Institute of Technology under IACUC protocol 1650.

The normalized photocurrent response was measured over 4 orders of magnitude by illuminating the tissue slice and recording the value of the steady state photocurrent as shown in Fig. 3(b). Fig. 3(c) shows a schematic of the setup used to record photocurrents and photopotentials with the patch pipette. Sample photocurrent and photopotential traces are shown in Fig. 3(d). The TRUE focusing technique enables a 30\% $(n=6)$ photocurrent enhancement factor, defined as the ratio between the difference between the photocurrent with TRUE focusing compared to the no shaping condition and the photocurrent for the no shaping condition. The no shaping condition was created by shifting the pattern 100 pixels in each direction on the SLM to maintain the background intensity but remove the TRUE focus. Similarly, the membrane voltage traces demonstrate that TRUE focusing can be used to modulate neuronal firing.

\section{a}

AAV-DJ-CaMKII-bReaChES-TS-YFP

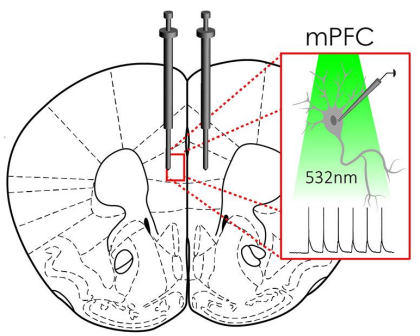

C

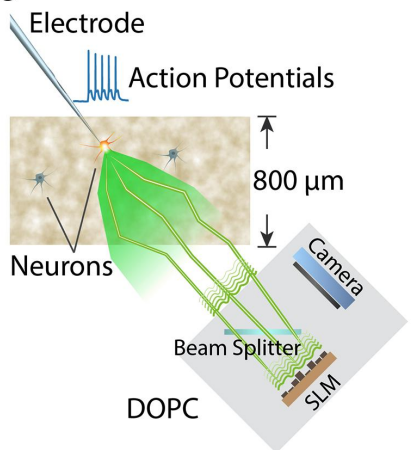

b

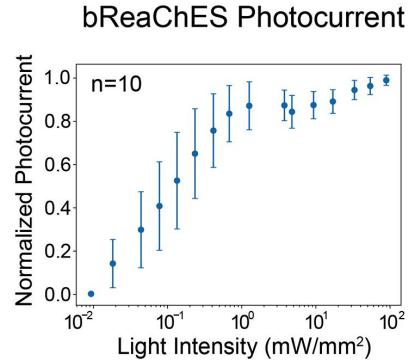

d

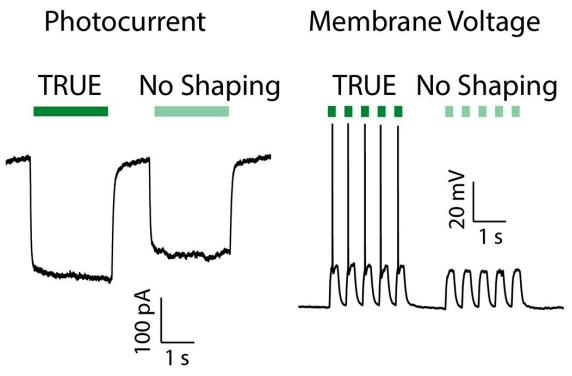

Figure 3. Opsin characterization and demonstration of optogenetic modulation via TRUE focusing (a) An AAV vector was used to sterotaxically deliver the bReaChES transgene into the mPFC. (b) The normalized photocurrent was measured with respect to light intensity to characterize the light response of the opsin. The average maximum photocurrent measured across the 10 cells studied was $1047 \mathrm{pA}$. (c) A diagram illustrating the experimental protocol used to demonstrate optogenetic modulation using TRUE focusing in $800-\mu \mathrm{m}$ thick living brain slices. (d) Representative photocurrent and membrane voltage traces with and without TRUE focusing. Reproduced from Reference 19.

Because the TRUE focus can maintain a focus profile that is dictated by the size of the ultrasound focus even when the tissue thickness increases beyond the optical diffusion limit, this enables improved resolution compared to conventional focusing. This improved resolution is demonstrated in Fig. 4. For conventional scanning, the input plane wave was tilted, generating a scanned focal point as shown in Fig. 4(a). To scan the TRUE focus, the ultrasound focus was raster scanned and the TRUE focusing procedure performed at each location to generate a TRUE focus. In each scanning configuration, the normalized photocurrent enhancement was measured at each point and used to generate a 2D scan map for conventional and TRUE focusing as shown in Fig. 4(c1) and (c2) respectively. Fitting each scan map with a 2D Gaussian function reveals FWHMs of 393 and $536 \mu \mathrm{m}$ in the $x$ and $y$ dimensions respectively for the conventional lens 
scan and FWHMs of 99 and $71 \mu \mathrm{m}$ in the $x$ and $y$ dimensions respectively for the TRUE focusing scan. The results of these scans show that TRUE focusing is able to achieve a nearly $4 \times$ higher lateral resolution compared to conventional focusing.
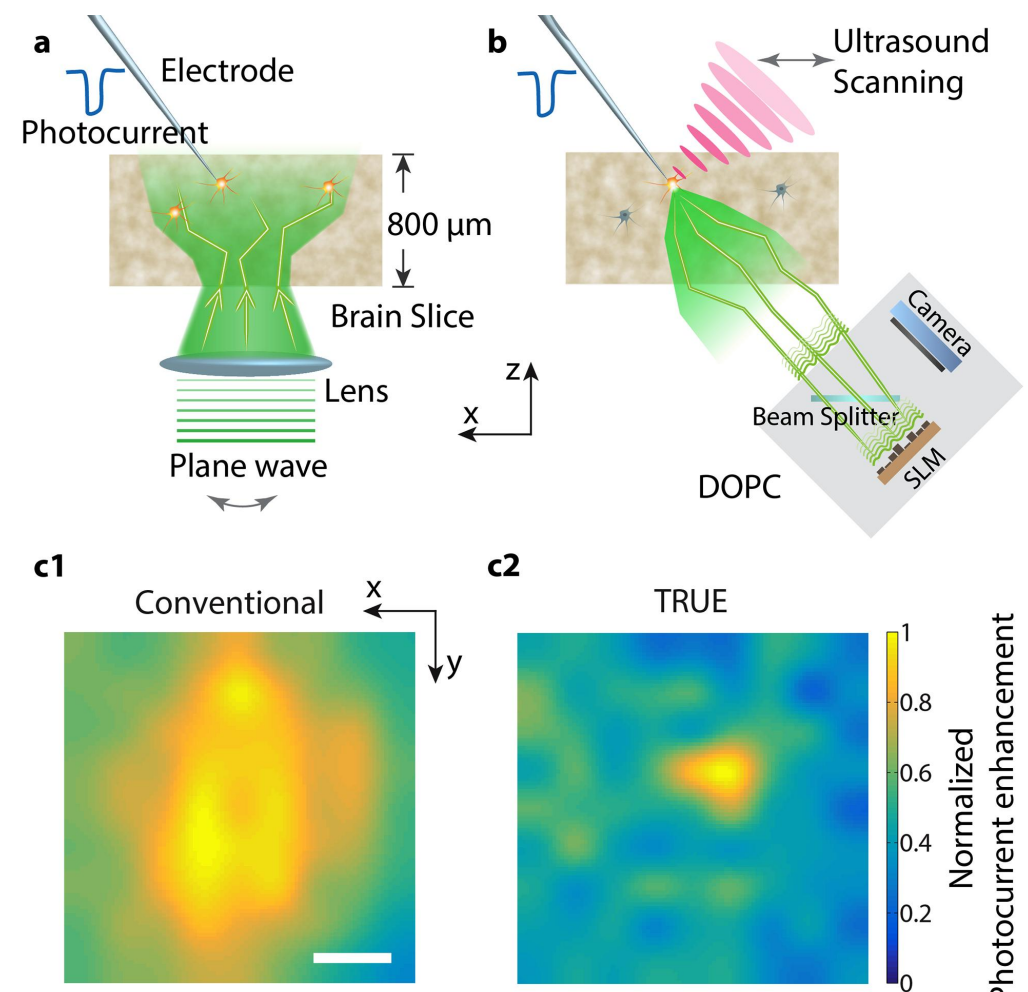

c2

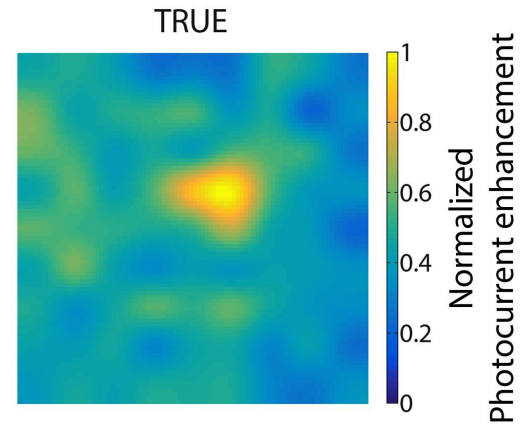

Figure 4. (a) Setup for scanning with conventional focusing. An input plane wave is tilted to scan the optical focus across the area surrounding a patch clamped neuron. (b) Setup for scanning with TRUE focusing. The ultrasound transducer is raster scanned through the slice and the TRUE focusing recording and playback procedure performed at each point to construct a 2D map of the photocurrent enhancement. (c) Photocurrent enhancement maps for conventional (c1) and TRUE (c2) focusing. Reproduced from Reference 19.

\section{CONCLUSIONS}

Developing methods to counteract scattering is important for improving the penetration depth of optical techniques. In particular, optogenetic modulation is an area of research where noninvasive methods for improved light delivery deep into tissue would help to improve the ability to study connections within the brain and move toward clinical applications. While techniques such as multiphoton microscopy can work noninvasively at depths of several hundred microns or more for neuromodulation, these methods are fundamentally limited by the number of unscattered photons which decreases exponentially with depth. In comparison, TRUE focusing enables multiply scattered photons to be harnessed to form optical foci.

The addressable depths demonstrated in this work were limited by the viability of cells located deep within the slices and the penetration depth of the DIC microscope which determined how deep neurons could be visualized for patching. While fluorescent indicators could in theory be used, these were not practical for our current experiments since the excitation wavelength for calcium indicators would have also excited neurons with opsins that match the 532-nm operating wavelength of the TRUE focusing system.

Future developments of the TRUE focusing technique include improving the guidestar modulation efficiency, improving the photocurrent enhancement, and increasing the operation speed of the TRUE focusing system. As TRUE focusing moves to deeper penetration depths, the amount of light which passes through the ultrasound focus decreases, in turn reducing the amount of ultrasound modulated light which is detected by the DOPC system. While recent work has 
shown that DOPC systems can operate even at very low light levels ${ }^{21}$, the signal-to-noise ratio still limits the ultimate depths accessible with the TRUE focusing system ${ }^{22}$.

Another area for improvement is the photocurrent enhancement factor. To improve this enhancement factor, the peak-tobackground ratio of the TRUE focusing system must be increased, either by increasing the number of modes controlled by the DOPC system or by reducing the number of modes within the ultrasound focus by shrinking its size.

Unfortunately, increasing the frequency of the ultrasound transducer to decrease the size of the focal zone is limited due to increasing ultrasound absorption at higher frequencies. Also, shrinking the size of the ultrasound focus below the size of a single cell may not help to increase the photocurrent enhancement factor since this will not increase the total energy delivered to the cell membrane. This leaves increasing the number of modes controlled by the DOPC system (i.e. the number of pixels on the camera and SLM) as the most feasible avenue for improvement. If the number of controllable modes of the DOPC system could be increased by a factor of 10 , this would improve the peak-to-background ratio of the TRUE focusing system to $\sim 12$, which would enable more practical applications across the field of wavefront shaping.

A final critical consideration for future development is operation speed. Moving toward in vivo applications it is important to account for how blood flow and other physiological motion such as breathing will impact the TRUE focusing system. Unfortunately, the phase conjugation procedure requires the scatterers within the sample to remain stationary between the recording and playback phases for successful focusing. In vivo, the movement of blood is the primary factor contributing to the movement of the scatterers and results in sub-millisecond decorrelation times at depths of only several millimeters ${ }^{23,24}$. Therefore, in order to successfully focus light at these depths, the TRUE focusing system must operate with a response speed shorter than the decorrelation time.

Unfortunately, as the number of pixels is scaled up to increase the peak-to-background ratio, attaining these operation speeds becomes even more challenging since the data must be transferred serially from the camera to a computing device and then transferred and displayed on the SLM. To solve this, we expect that integrated wavefront sensing and shaping devices will be necessary where each individual pixel acts as an independent capture, calculation, and modulation unit ${ }^{25}$. This will allow for the wavefront shaping procedure to be parallelized and enable the number of controllable modes to be increased in a scalable way without sacrificing speed. Such a device would also greatly reduce technical barriers (e.g., the difficult alignment procedure) which prevent the widespread adoption of wavefront shaping technology and enable scientists to more broadly incorporate wavefront shaping methods into their optical technologies.

\section{REFERENCES}

[1] Ntziachristos, V., "Going deeper than microscopy: the optical imaging frontier in biology," Nat. Methods 7(8), 603-614 (2010).

[2] Helmchen, F. and Denk, W., "Deep tissue two-photon microscopy," Nat. Methods 2(12), 932-940 (2005).

[3] Vellekoop, I. M. and Mosk, A. P., "Focusing coherent light through opaque strongly scattering media," Opt. Lett. 32(16), 2309-2311 (2007).

[4] Vellekoop, I. M., Cui, M. and Yang, C., "Digital optical phase conjugation of fluorescence in turbid tissue.," Appl. Phys. Lett. 101(8), 81108 (2012).

[5] Zhou, E. H., Ruan, H., Yang, C. and Judkewitz, B., "Focusing on moving targets through scattering samples," Optica 1(4), 227-232 (2014).

[6] Ma, C., Xu, X., Liu, Y. and Wang, L. V., "Time-reversed adapted-perturbation (TRAP) optical focusing onto dynamic objects inside scattering media," Nat. Photonics 8(12), 931-936 (2014).

[7] Kong, F., Silverman, R. H., Liu, L., Chitnis, P. V, Lee, K. K. and Chen, Y. C., "Photoacoustic-guided convergence of light through optically diffusive media," Opt. Lett. 36(11), 2053 (2011).

[8] Caravaca-Aguirre, A. M., Conkey, D. B., Dove, J. D., Ju, H., Murray, T. W. and Piestun, R., "High contrast three-dimensional photoacoustic imaging through scattering media by localized optical fluence enhancement," Opt. Express 21(22), 26671 (2013).

[9] Chaigne, T., Katz, O., Boccara, A. C., Fink, M., Bossy, E. and Gigan, S., "Controlling light in scattering media non-invasively using the photoacoustic transmission matrix," Nat. Photonics 8(1), 58-64 (2014).

[10] Lai, P., Wang, L., Tay, J. W. and Wang, L. V., "Photoacoustically guided wavefront shaping for enhanced optical focusing in scattering media," Nat. Photonics 9(2), 126-132 (2015). 
[11] Tay, J. W., Lai, P., Suzuki, Y. and Wang, L. V., "Ultrasonically encoded wavefront shaping for focusing into random media.," Sci. Rep. 4, 3918 (2014).

[12] Xu, X., Liu, H. and Wang, L. V., "Time-reversed ultrasonically encoded optical focusing into scattering media," Nat. Photonics 5(3), 154-157 (2011).

[13] Wang, Y. M., Judkewitz, B., DiMarzio, C. a. and Yang, C., "Deep-tissue focal fluorescence imaging with digitally time-reversed ultrasound-encoded light," Nat. Commun. 3, 928 (2012).

[14] Si, K., Fiolka, R. and Cui, M., "Fluorescence imaging beyond the ballistic regime by ultrasound pulse guided digital phase conjugation.," Nat. Photonics 6(10), 657-661 (2012).

[15] Ruan, H., Jang, M. and Yang, C., "Optical focusing inside scattering media with time-reversed ultrasound microbubble encoded light," Nat. Commun. 6, 1-8 (2015).

[16] Ruan, H., Haber, T., Liu, Y., Brake, J., Kim, J., Berlin, J. M. and Yang, C., "Focusing light inside scattering media with magnetic-particle-guided wavefront shaping," Optica 4(11), 1337 (2017).

[17] Boyden, E. S., Zhang, F., Bamberg, E., Nagel, G. and Deisseroth, K., "Millisecond-timescale, genetically targeted optical control of neural activity.," Nat. Neurosci. 8(9), 1263-1268 (2005).

[18] Warden, M. R., Cardin, J. A. and Deisseroth, K., "Optical Neural Interfaces," Annu. Rev. Biomed. Eng. 16(1), 103-129 (2014).

[19] Ruan, H., Brake, J., Robinson, J. E., Liu, Y., Jang, M., Xiao, C., Zhou, C., Gradinaru, V. and Yang, C., “Deep tissue optical focusing and optogenetic modulation with time-reversed ultrasonically encoded light," Sci. Adv. 3(12), eaao5520 (2017).

[20] Kim, C. K., Yang, S. J., Pichamoorthy, N., Young, N. P., Kauvar, I., Jennings, J. H., Lerner, T. N., Berndt, A., Lee, S. Y., Ramakrishnan, C., Davidson, T. J., Inoue, M., Bito, H. and Deisseroth, K., "Simultaneous fast measurement of circuit dynamics at multiple sites across the mammalian brain," Nat. Methods 13(4), 325-328 (2016).

[21] Jang, M., Yang, C. and Vellekoop, I. M., "Optical Phase Conjugation with Less Than a Photon per Degree of Freedom," Phys. Rev. Lett. 118(9), 1-5 (2017).

[22] Liu, Y., Ma, C., Shen, Y., Shi, J. and Wang, L. V., "Focusing Light Inside Scattering Media with Optical Phase Conjugation," Optica 4(2), 280-288 (2016).

[23] Liu, Y., Lai, P., Ma, C., Xu, X., Grabar, A. A. and Wang, L. V., "Optical focusing deep inside dynamic scattering media with near-infrared time-reversed ultrasonically encoded (TRUE) light," Nat. Commun. 6, 5904 (2015).

[24] Qureshi, M. M., Brake, J., Jeon, H.-J., Ruan, H., Liu, Y., Safi, A. M., Eom, T. J., Yang, C. and Chung, E., "In vivo study of optical speckle decorrelation time across depths in the mouse brain," Biomed. Opt. Express 8(11), 4855 (2017).

[25] LaForest, T., Verdant, A., Dupret, A., Gigan, S., Ramaz, F., Tessier, G. and Benoit à la Guillaume, É., “Cointegration of a smart CMOS image sensor and a spatial light modulator for real-time optical phase modulation," Proc. SPIE 9022, 90220N (2014). 